Research and Practice in Technology Enhanced Learning

(C) World Scientific Publishing Company \&

Asia-Pacific Society for Computers in Education

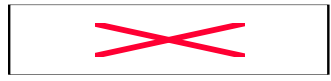

\title{
APPLYING WEB PAGE ADAPTATION TECHNIQUE TO THE AUGMENTATION OF MOBILE LEARNING
}

\author{
Stephen J.H. Yang \\ Department of Computer Science and Information Engineering, National Central University, No. 300, Jhongda \\ Rd., Jhongli City, Taoyuan County 32001, Taiwan \\ jhyang@csie.ncu.edu.tw \\ Jia Zhang \\ Department of Computer Science, Northern Illinois University, 1425 W. Lincoln Hwy., DeKalb, IL 60115-2825 \\ jiazhang@cs.niu.edu \\ Angus F.M. Huang \\ Department of Computer Science and Information Engineering, National Central University, No. 300, Jhongda \\ Rd., Jhongli City, Taoyuan County 32001, Taiwan \\ fmhuang@csie.ncu.edu.tw
}

\begin{abstract}
The goal of this research is to study how to augument mobile learning by applying Web page adaptation techniques. In this paper, we present a case study of how we applied Web page adaptation to facilitate mobile learning on the Blackboard Learning System. Without requiring different versions of the original learning materials, our research provides automatic delivery and presentation of adaptive Web-based learning materials based on students' receiving profiles. Experimental results demonstrate that our method provides effective and efficient delivery of Web-based learning material over the mobile Internet, which results in an extension of the time and space of learning, as well as encouraging and facilitating collaboration among students.
\end{abstract}

Keywords: Learning; Mobile Internet; Handheld Devices; Web Page Presentation; Learning Material.

\section{Introduction}

National Central University (NCU) deployed the Blackboard Learning System (or BB in short throughout the paper) (Blackboard.com) in early 2004 to build a centralized repository of course materials and to enable online learning. However, the initial adoption of BB was quite low. At the end of 2004, the number of BB courses offered was 42; the number of teachers who adopted BB as a supporting teaching environment was 14 .

The ultimate goal of this project is to increase BB usage at NCU, in terms of the number of students, teachers, and courses. Particularly, as students increasingly use various types of handheld devices (or so-called mobile devices such as PDAs and mobile phones) to access the Internet, this project aims to promote mobile learning on $\mathrm{BB}$. A number of efforts applying the mobile and wireless technologies to school administration, 
classroom activity, and exam management have been proposed and received significant momentum in recent years. In contrast with these works focusing on applying handheld devices to facilitate and enhance mobile learning and collaborative learning, our research focuses on automatic Web page adaptation to support Web page delivery for mobile learning specific to BB. By Web page adaptation, we refer to a "multiserving" model from the same Web content. Without requiring development of different versions of the original learning materials, our research provides automatic content adaptation and delivery of adaptive Web-based learning materials based on students' receiving situations (e.g., devices).

The necessity of using Web page adaptation to support learning over the mobile Internet is illustrated in Figure 1. The left-hand side of the figure is the original desktopbased Web page display (layout and content), and the right-hand side of the figure is the adapted page based on a PDA screen. In order to adapt the Web page originally designed for a desktop (on the left) and view it on a PDA (on the right), the font and size of the images, texts, and hyperlinks need to be resized, and the layout also needs to be reformatted into a single column (the original multi-column horizontal layout is transformed into a vertical layout) to fit into a PDA's small screen.

In our previous research, we created a dynamic mechanism that automatically analyzes and adapts HTML Web content based on receivers' contextual situations and requirements (Yang, Zhang et al. 2007c). In this project, we explore how to apply and exploit our Web page adaptation technique to improve learning material accessibility on the mobile Internet, while maintaining semantic coherence of the presentation objects in original Web pages.

After applying our technique, the adoption of BB at NCU has been greatly increased. During the three-year period from Fall 2004 to Spring 2007, the number of BB courses offered has increased from 42 to 677 , which counts for about $48 \%$ of the total courses offered at NCU. The number of teachers who adopt BB as a supporting teaching environment has increased from 14 to 336, which counts for about $60 \%$ of the total number of teachers employed by NCU. The number of students who use BB as a supporting learning environment has increased from 495 to 7968 , which counts for about $68 \%$ of the total students enrolled in NCU.

The remainder of the paper is organized as follows. We will first discuss mobile learning with handheld devices over the mobile Internet. Then we will explain how we apply our dynamic Web page adaptation technique to promote mobile learning. Afterwards, we will present the details of our conducted experiments and discussions. Finally, we will make conclusions.

\section{Handheld Devices: What, Why, Where, And When To Use?}


The above discussions emphasize the importance and popularity of adopting handheld devices to enable and facilitate mobile learning. In this section, we report an experiment that we conducted to explore the necessity of applying the Web page adaptation

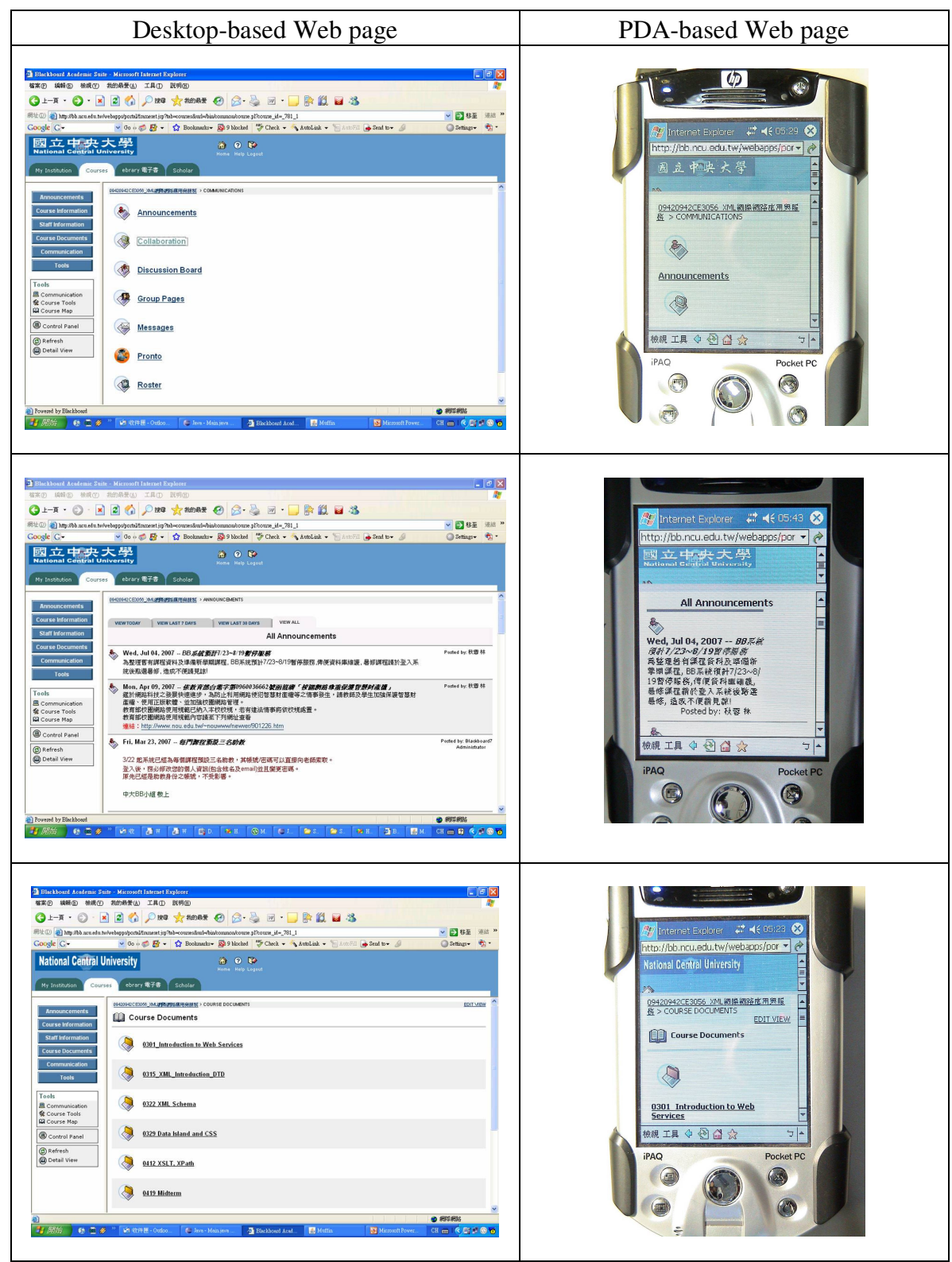

Figure 1. Comparison of browsing BB using desktop and PDA. 
technique in mobile learning with handheld devices. Meanwhile, these test data are used as baselines to validate the results of applying our dynamic Web page adaptation mechanism later on. We invited 30 students and asked them to browse non-adapted learning materials with notebooks (be abbreviated as NB), PDAs, and mobile phones. As shown in Table 1, we recorded each student's browsing satisfaction with every type of learning device. Students indicated their degree of satisfaction on scales of 0 to 100 . We collected these data and used one-way repeated measures ANOVA to compare the variation of satisfaction among the three types of learning devices. The statistic results shown in Table 2 and Table 3 indicate that the influence of different kinds of handheld devices to the learners' satisfaction is significant. According to the analysis, we can see that NB is considered the best device for Web browsing. In order to let learners have the same browsing experience with PDAs and phones as they are with NBs, content adaptation is a critical technique in mobile learning.

Table 1. Degree of satisfaction regarding the browsing experience on three types of handheld devices (30 students).

\begin{tabular}{|c|c|c|c|c|c|c|c|c|}
\hline \multicolumn{3}{|c|}{ NB } & \multicolumn{3}{|c|}{ PDA } & \multicolumn{3}{|c|}{ Phone } \\
\hline 94.00 & 94.00 & 87.00 & 88.00 & 87.00 & 78.00 & 85.00 & 76.00 & 79.00 \\
\hline 92.00 & 97.00 & 93.00 & 78.00 & 84.00 & 88.00 & 77.00 & 75.00 & 79.00 \\
\hline 93.00 & 92.00 & 96.00 & 85.00 & 86.00 & 81.00 & 83.00 & 85.00 & 80.00 \\
\hline 90.00 & 92.00 & 94.00 & 82.00 & 86.00 & 80.00 & 75.00 & 84.00 & 80.00 \\
\hline 95.00 & 89.00 & 94.00 & 81.00 & 82.00 & 79.00 & 74.00 & 86.00 & 82.00 \\
\hline 86.00 & 95.00 & 96.00 & 82.00 & 85.00 & 84.00 & 65.00 & 83.00 & 69.00 \\
\hline 92.00 & 96.00 & 93.00 & 85.00 & 80.00 & 85.00 & 70.00 & 80.00 & 84.00 \\
\hline 90.00 & 96.00 & 97.00 & 86.00 & 80.00 & 85.00 & 80.00 & 84.00 & 75.00 \\
\hline 87.00 & 90.00 & 97.00 & 77.00 & 84.00 & 86.00 & 71.00 & 80.00 & 72.00 \\
\hline 91.00 & 92.00 & 94.00 & 86.00 & 89.00 & 82.00 & 82.00 & 86.00 & 65.00 \\
\hline \multicolumn{3}{|c|}{$\bar{X}_{\mathrm{NB}}=92.800$} & \multicolumn{3}{|c|}{$\bar{X}_{\mathrm{PDA}}=83.367$} & \multicolumn{3}{|c|}{$X_{\text {Phone }}=78.200$} \\
\hline \multicolumn{9}{|c|}{$\bar{X}_{\mathrm{all}}=84.789$} \\
\hline
\end{tabular}

Table 2. Estimations of students' degrees of satisfaction regarding their Web browsing experience on three types of handheld devices.

\begin{tabular}{|l|l|l|l|l|}
\hline Satisfaction & Mean & \multirow{2}{*}{$\begin{array}{l}\text { Standard } \\
\text { Error }\end{array}$} & \multicolumn{3}{|l|}{ 95\% Confidence Interval } \\
\cline { 4 - 5 } & & & Lower Bound & Upper Bound \\
\hline NB & 92.800 & 0.556 & 91.663 & 93.937 \\
PDA & 83.367 & 0.594 & 82.152 & 84.582 \\
Phone & 78.200 & 1.100 & 75.950 & 80.450 \\
\hline
\end{tabular}


To better understand what students thought about handheld devices and how Web page adaptation may affect students' usages on handheld devices, we surveyed 87 freshmen enrolled at the department of computer science and information engineering in the Fall 2007 semester and received responses from 56 students. The survey questions and results are presented as follows.

Table 3. Pairwise comparisons of satisfaction regarding the Web browsing experience on three types of handheld devices.

\begin{tabular}{|c|c|r|r|r|r|r|}
\hline \multirow{2}{*}{$\begin{array}{c}\text { Satisfaction } \\
\text { Satisfaction }\end{array}$} & $\begin{array}{c}\text { Mean } \\
\text { Difference } \\
(\mathrm{I}-\mathrm{J})\end{array}$ & $\begin{array}{c}\text { Standard } \\
\text { Error }\end{array}$ & Sig. & & \multicolumn{2}{|c|}{$\begin{array}{c}\text { 95\% Confidence } \\
\text { Interval for Difference }\end{array}$} \\
\cline { 5 - 7 } & & & & $\begin{array}{c}\text { Lower } \\
\text { Bound }\end{array}$ & $\begin{array}{c}\text { Upper } \\
\text { Bound }\end{array}$ \\
\hline NB & PDA & $9.433^{*}$ & 0.725 & .000 & 7.950 & 10.916 \\
& Phone & $14.600^{*}$ & 1.206 & .000 & 12.134 & 17.066 \\
\hline PDA & NB & $-9.433^{*}$ & 0.725 & .000 & -10.916 & -7.950 \\
& Phone & $5.167 *$ & 1.116 & .000 & 2.884 & 7.450 \\
\hline Phone & NB & $-14.607^{*}$ & 1.206 & .000 & -17.066 & -12.134 \\
& PDA & $-5.167 *$ & 1.116 & .000 & -7.450 & -2.884 \\
\hline
\end{tabular}

Based on estimated marginal means,

*. The mean difference is significant at the .05 level.

a. Adjustment for multiple comparisons: Least Significant Difference (equivalent to no adjustment).

The survey consists of five questions to analyze students' favorite type of handheld devices, their usage patterns, and mostly expected services.

(1) What kinds of handheld devices do you use (list multiple devices if necessary)?

(2) Why do you use handheld devices (list multiple reasons if necessary)?

(3) Where do you use handheld devices most?

(4) When do you use handheld devices most?

(5) Which services do you use most and least on your handheld devices?

By analyzing the survey results, we found that cellular phone is the most popular handheld device that the students use to access the mobile Internet, and mobility and convenience are the two top reasons why they use handheld devices (see Figure 2). 


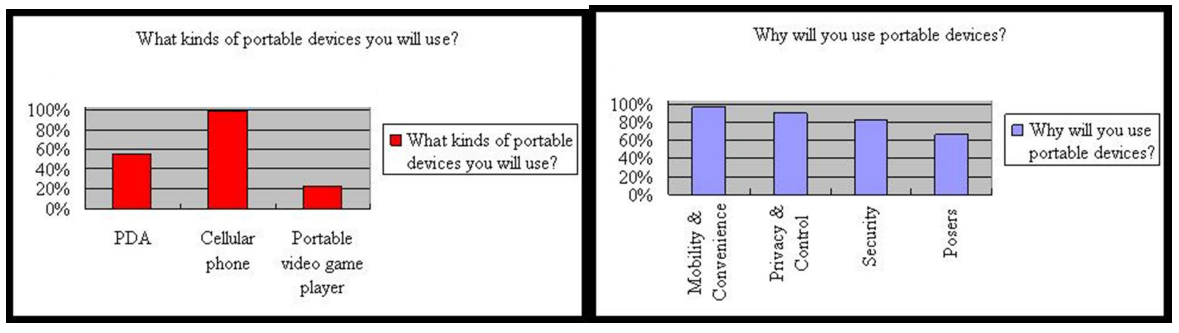

Figure 2. What kinds of handheld devices most students use and why.

We also found that most students prefer to use their handheld devices in public areas or when they have a little free time such as waiting for bus or during their lunch break at café (see Figure 3). Students seldom use handheld devices when usages of such devices are prohibited (such as during meetings or driving)_ or at places where they have access to desktop computers (such as at office and at home).

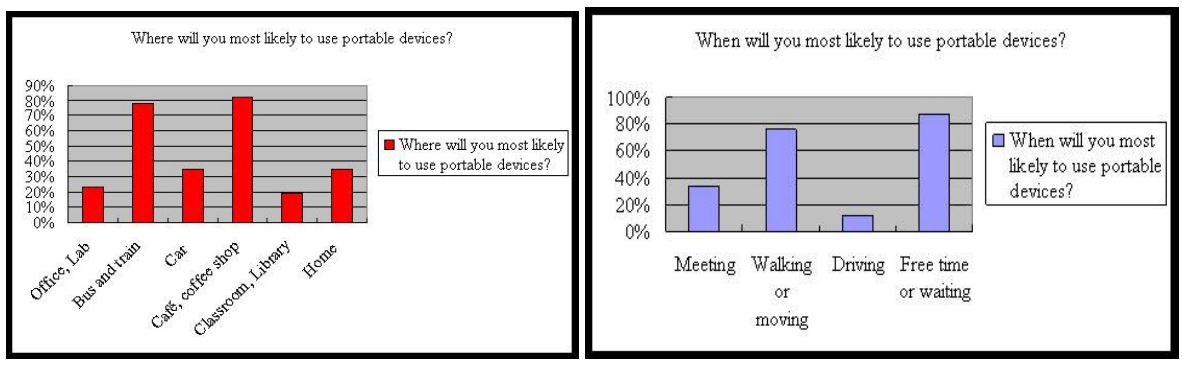

Figure 3. Where and when most students use handheld devices.

The top three types of most desired services on handheld devices are summarized as follows: first is media playing service for music and movies; second is Web browsing service; third is communication service such as phone service, instant messenger service, and email service (see Figure 4). We also found that students do not use handheld devices for word processing and their homework. Furthermore, we found that many students do not keep their profiles on their devices due to privacy concerns.

Based on the aforementioned findings, we conclude that most of the students like to remain connected on the Internet with mobile phones or PDAs when they are free at public areas. Nevertheless, students prefer to leave document processing and heavy computing work to conventional computing devices such as desktop and notebook computers. Therefore, one of the crucial factors discouraging students from learning over the mobile Internet is the limitation of handheld devices. In most cases, handheld devices are featured with limited computing powers, small-size screens and changing conditions (e.g., a student may try to access Web pages while moving). This divergence increases 
the difficulty of presenting user-friendly Web pages for all kinds of devices. Furthermore, most existing Web pages are designed for displaying on desktop computers. Their default settings and style-sheets (e.g., image sizes, font sizes, and layout structures) may not be suitable to be presented on portable devices. As a result, a technique is needed to deliver adaptive Web pages in any format to any device through any network at anytime from anywhere. Considering that a learning material typically undergoes frequent changes, the conventional approach of preparing two versions (formats) of the same learning material, one for desktops and one for handheld devices, is neither practical nor feasible for Web learning material delivery.

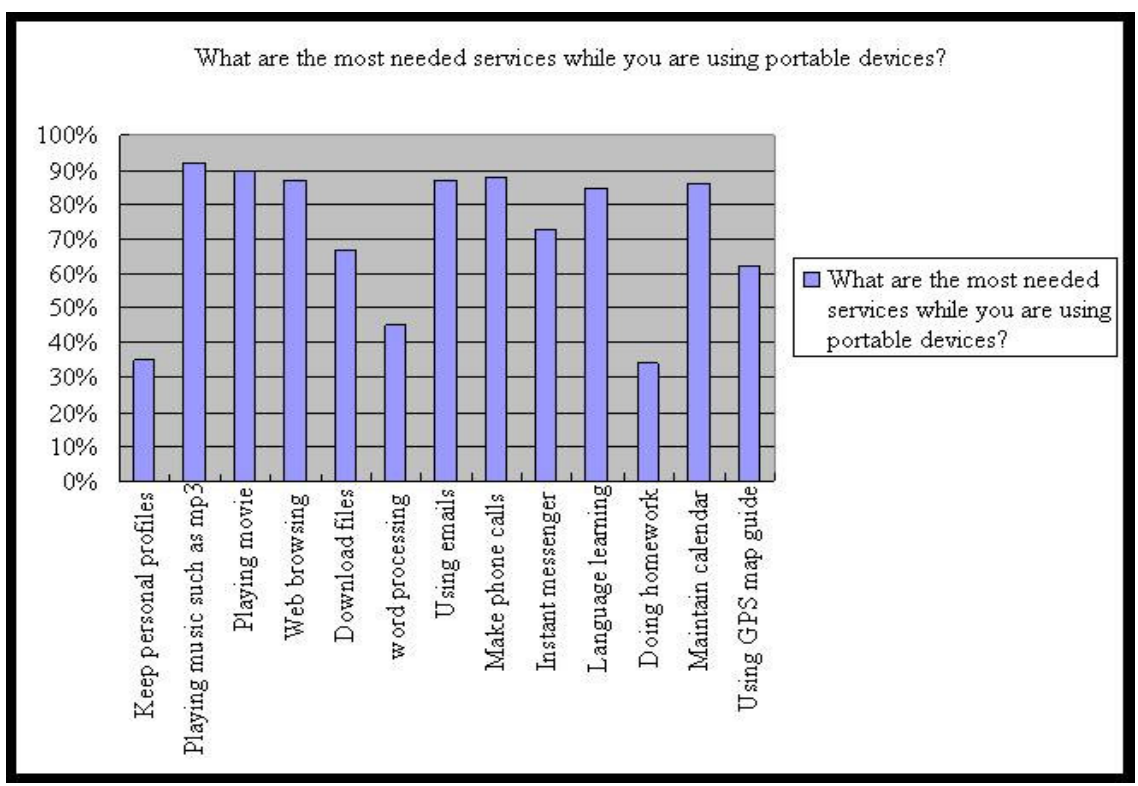

Figure 4. Services students use on handheld devices.

\section{Related Work}

Applying the mobile and wireless technologies to school administration, classroom activity, and exam management have been proposed and received significant momentum in recent years. For example, AL-Bastaki and Al-Ajeeli (2005) present a Wireless Application Protocol (WAP)-based course registration system that is designed and implemented to facilitate students' registration process. Their system applies WAP-based technology to provide a variety of services such as wireless commerce, cashless payment, and location-based services. Markett, Sánchez, Weber, \& Tangney (2006) utilize mobile phones and short message service (SMS) within a classroom. Their research allows students to send real-time SMS in the middle of a class using their personal mobile 
phones. Exploiting a modem interfacing with customized software to produce SMS files, a teacher can view messages and verbally develop an interactive loop with students during a class. Segall, Doolen, \& Porter (2005) indicate that one of the most common usages of PDAs in a classroom is as a test administrator. Their study compares the usability effectiveness, efficiency, and satisfaction of a PDA-based quiz application to that of a standard paper-and-pencil quiz in a university course. They found no difference in effectiveness and satisfaction levels between the two quiz types, implicating that computer anxiety was not affected by the quiz type. For these reasons, as well as other advantages to both students (e.g., real-time scoring) and teachers (e.g., less time spent on grading), PDAs have been considered as an attractive test administration option for schools and universities.

Research on utilizing handheld devices for supporting collaborative learning, student model building, and intelligent tutoring systems has also been proposed to extend the usage of mobile technology. Zurita and Nussbaum (2004) propose an effective way of using handheld computers to support collaborative learning activities in elementary classrooms. They found that many weaknesses of applying technology in collaborative learning (e.g., coordination, communication, organization of materials, negotiation, interactivity, and lack of mobility) can be solved with a mobile computer-supported collaborative learning environment with handheld devices interconnected by a wireless network. Chang, Wang, \& Chen (2006) develop a ubiquitous learning website that provides functions enabling learning to take place anytime and anywhere with any available learning device. They record learners' behaviors over the website as learning portfolios and analyze for behavioral diagnosis or instructional planning. Based on the recorded learning portfolios, they build a student model and a concept map of the learning domain for the development of a ubiquitous learning environment to enhance learning performance via learning status awareness, schedule reminders and mentor recommendation. Virvou and Alepis (2005) indicate that one of the significant contributions of mobile technology to education is enabling students and instructors to make constructive use of their spare time to work on lessons at any place, even away from offices, classrooms, and laboratories where computers are usually located. They also develop a mobile authoring tool called Mobile Author, which can be used by human instructors either from a computer or a mobile phone to create their own Intelligent Tutoring Systems (ITSs) and to distribute the ITSs to their students. Students can also use any computer or mobile phone to have access to the ITSs. The tutoring systems can assess students' performance, record student progress, and provide advice adapted to the needs of individual students.

These efforts indicate that mobile devices have become increasingly important to be used to support and supplement traditional teaching and learning. However, their flexibility is not without penalty since the value added by this new trend can be largely defeated if we cannot ensure that content be properly displayed on mobile devices. In contrast with these works focusing on applying handheld devices to facilitate and 
enhance mobile learning and collaborative learning, our research focuses on automatic Web page adaptation to support Web page delivery in mobile learning specific to BB.

Some of our previous work is closely related to this paper. In (Yang, Zhang et al. 2007a), we present a JESS-enabled context elicitation system featuring an ontologybased context model to formally describe and acquire contextual information pertaining to service requesters and Web services. In (Yang, Zhang et al. 2007c), we present a Unit Of Information (UOI)-based content adaptation method, which automatically detects semantic relationships among comprising components in Web content, and then reorganizes page layout to fit handheld devices based on identified UOIs. In (Yang, Zhang et al. 2007b), we present an ontology-based context model supported by context query and phased acquisition techniques. In contract to those reports focusing on generic Web content adaptation algorithms, this paper focuses on how we apply our generic Web page adaptation mechanism as the underlying engine supporting mobile learning.

\section{Dynamic Web Page Adaptation on the Mobile Internet}

In this section, we present how we apply dynamic Web page adaptation to deliver learning materials on the mobile Internet. The conventional approach of providing the same Web page content for different handheld devices is to prepare specific versions (formats) of the same content for specific mobile devices. For example, a Web page holds one HTML version supporting desktop devices and one Wireless Markup Language (WML) version supporting wireless devices. This approach is straightforward but laborintensive yet inflexible. Web content providers have to prepare different layouts and formats for the same Web content, which results in tremendous overhead. Even worse, any change in the content may result in consequent changes in every related version, which is highly inflexible and may easily cause inconsistency. Considering a course material typically undergoes frequent changes, this traditional approach is neither practical nor feasible for course content delivery.

In our continuous research of Web page adaptation (Yang and Shao 2006; Yang, Zhang et al. 2007c), we have investigated and summarized five Web page adaptation techniques: resizing, column-wise, thumbnail, replacing, and transcoding. Resizing is a technique to reduce content's shape to fit within the smaller screen size of portable devices. It allows people to view content as much as possible in a small screen size. Column-wise is a technique to transform content's presentation layout from multiplecolumns to a single-column, while reducing the sizes of contained images and text if necessary. Column-wise reduces users' movement of horizontal bars. Thumbnail is a technique to replace a large area of image with small icons to fit within a smaller screen size, while saving memory and transmission bandwidth. Replacing is a technique to replace rich media with text or voice (with the same meaning) in order to save space and bandwidth, and create an alternative when some types of media cannot be played on portable devices. Transcoding is a technique to transform media types with different modalities and fidelity to fit in with individual portable device's computing needs. 
Transcoding is also used to reduce the use of memory as well as the transmission bandwidth, especially when portable devices have less computing power.

Resizing and column-wise transformation of the original learning content from a multiple-column layout into a single-column one provides better readability without losing meaningful information. Thumbnail, replacing, and transcoding share the similar concept of transforming image and media into corresponding modality and fidelity to overcome the memory, computing, and communication limitation of portable devices. In most cases, resizing and column-wise are the simplest page adaptation solutions. Nevertheless, they also introduce severe problems. Without retaining semantic coherence and relationships between semantic units, a simple page adaptation may mess up the organization of a page and lead to misunderstanding.

\subsection{Web page adaptation}

Our Web page adaptation technique describes and manages students' and devices' profiles based on Composite Capabilities/Preferences Profile (CC/PP) recommended by the W3C and User Agent Profile (UAProf) recommended by the WAP Forum. We implement Web page adaptation with an open-source software: use Deli as a CC/PP parser to formalize students' and devices' profiles, and use Cocoon as an adaptation engine to prepare adaptive Web pages based on formalized students' and devices' profiles. Figure 5 shows the architecture of our Web page adaptation. This architecture is based on the concept of creating Web page once and then using it for various types of presentation. Instead of using HTML, this architecture uses XML and XSL to describe Web pages and their presentation layouts, respectively. Cocoon is the adaptation engine implemented with XSLT, which can generate various documents such as SMIL, XHTML, SNG, PDF based on XML and XSL. 


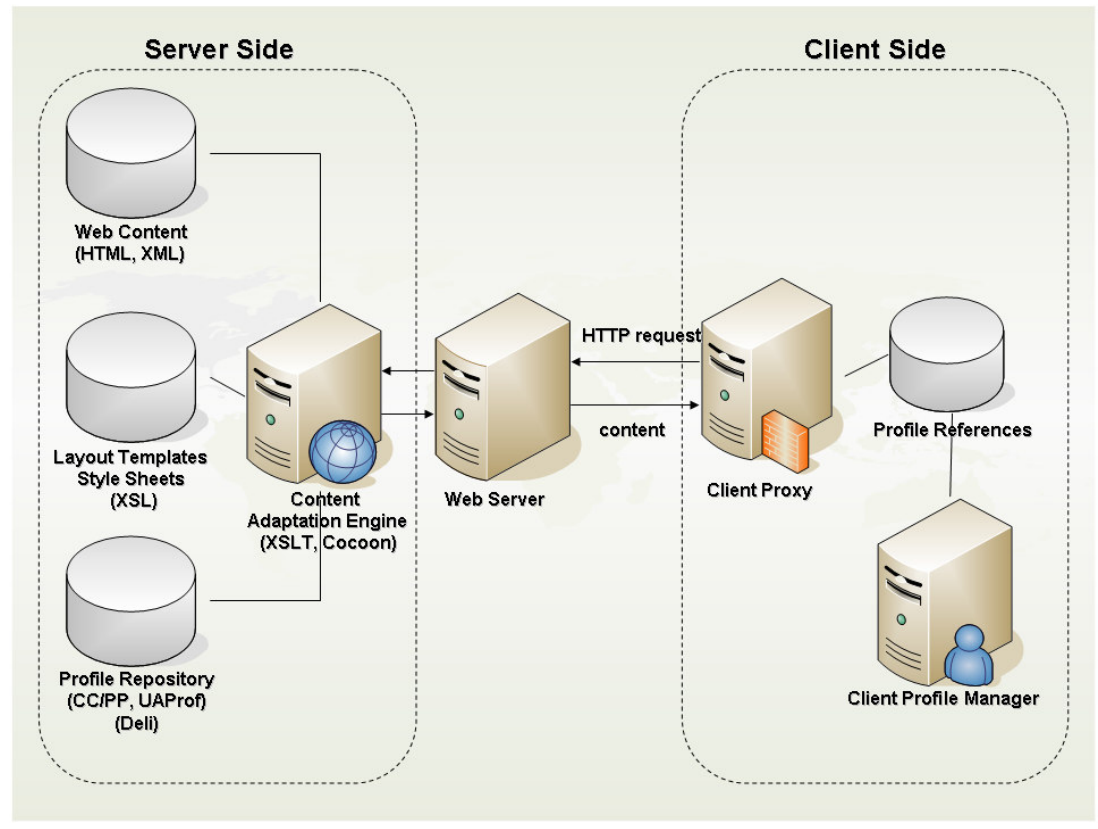

Figure 5. Architecture for Web page adaptation.

\subsection{Profile description and profile resolution}

It is necessary to understand students' computing contexts before performing Web page adaptation. Ideally, such contextual information can be described by students' and devices' profiles and can be stored at the server side. As shown in Figure 5, Web page adaptation is achieved by using profile description and profile resolution. The server side stores students' and devices' profile descriptions in the profile repository, and the client side keeps profile references in the profile repository. Instead of sending an entire profile with every request, a client only sends a reference to the profile repository. The process of interpreting the profile references is known as profile resolution. The client proxy acts as an HTTP proxy by inserting profile headers describing client's device profile. In the CC/PP framework, a client device sends its CC/PP profile to an adaptation server through HTTP headers during an HTTP request. The adaptation engine on the server side then uses the profile to transform the requested Web page. The client profile manager consists of a library of APIs that can be used to change or add attributes and values to the profile repository. The manager can automatically monitor various client devices' states and other operational characteristics that affect the clients' profiles. (e.g. battery status, screen contrast, network bandwidth, etc.).

Since most of Internet-based learning is presented in Web pages, the design of our Web page adaptation includes three phases - Web page decomposition, dynamic 
transcoding, and adapted Web page rendering. A Web page comprises a set of presentation objects, which are characterized by modality indicating objects' types (e.g., text, video, audio, and image). Each modality is associated with fidelity indicating objects' quality such as image resolution, color depth, and video bit-rate. In order to render the same object on various devices, Web page adaptation may have to perform transcoding to change object's modality and fidelity.

\subsection{Web page decomposition}

The Web page adaptation begins with Web page decomposition. We first transform a non-well-formed Web page into a well-formed format using an open software tool: Tidy (http://tidy.sourceforge.net/). The objects encompassed in a Web page can be decomposed and identified based on W3C's DOM (http://www.w3.org/DOM/). The result of Web page decomposition is a set of decomposed objects, which will be stored in an object repository.

Besides identifying individual objects, inter-object relationships can also be identified during Web page decomposition. Relationships such as spatial and temporal presentation sequence can be used to describe the layout and sequence of objects when we do adapted Web page rendering. For example, two objects that were rendered side by side spatially in a NB's screen could be adapted to be displayed sequentially from top to bottom on a mobile phone's screen.

In order to identify and record inter-object relationships, we construct an object tree for each decomposed Web page to maintain objects' spatial and temporal relationships. This study focuses on dealing with the web pages in html-based documents at present. Therefore, we use the html tags to recognize the information within web pages. The contents annotated in the same tag tuple, e.g. $<$ table $><$ table $>$ or $<$ a href="'" $></$ a $>$, will form a specific semantic area. We define them as objects and their typesetting sequence as inter-object relationships. Objects can be displayed individually or aggregated into a segment. The root of an object tree (as shown in Figure 6) represents a Web page, the intermediate nodes represent segments, and the leaf nodes represent individual objects. An object tree can be traversed in a linear sequence with the forms of pre-order or inorder relation. This linear sequence will be used to restore inter-object relationship when we do Web page rendering. 


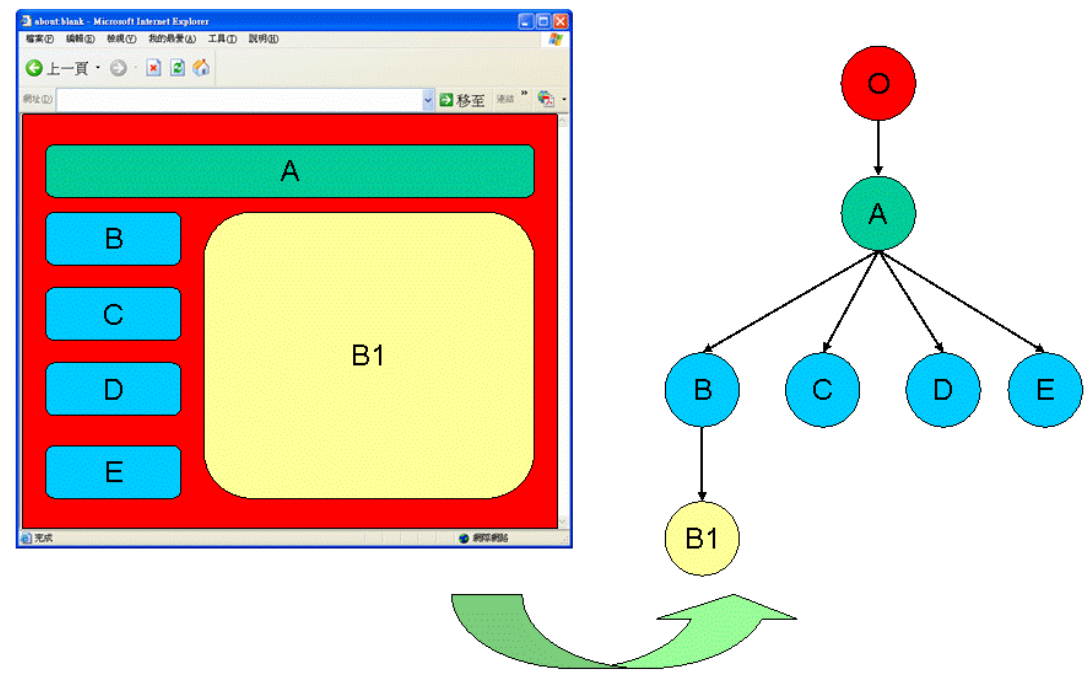

Figure 6. Example of a decomposed Web page and its corresponding object tree.

\subsection{Dynamic trans-coding}

We have utilized various third-party transcoding tools to transform objects into various modality and fidelity. The transcoded objects will be cached and then stored into an object repository. Many third-party transcoding tools have been used in this research, including VCDGear (www.vcdgear.com) which is a toolbox for video transformation, PictView (http://www.pictview.com/pvw.htm) which is a command-line driven image converter, and JafSoft (http://www.jafsoft.com/asctohtm/) and Microsoft Reader (http://www.microsoft.com) which are text to speech (and vice versa) converters. With this approach of using third-part transcoding services, any newly developed tools can be easily incorporated into the system to enhance adaptation performance.

For dynamic Web page adaptation, we need to perform dynamic transcoding and caching when applicable objects cannot be found. The advantage of dynamic Web page adaptation provides the flexibility that allows students to access any Web page and obtain adapted Web page upon their requests. The challenge is that the performance will degrade due to additional transcoding overhead. Students probably have to wait a long time when they access adapted Web page the first time. Nevertheless, the performance will become better the next time when the same student and other students access the same adapted Web page. The reason is that we have applied a caching mechanism so that the transcoding overhead can be reduced. Instead of caching the entire adapted Web page, only the dynamically transcoded objects will be cached. When dynamic Web page adaptation begins, the dynamically generated layout template and the associated objects 
will also be cached for dynamic page rendering. This is because the size of the transcoded objects have been reduced, which results in tremendous saving of transmission bandwidth.

\subsection{Adapted Web page rendering}

Adapted Web page rendering is designed to dynamically aggregate an XSL-based layout template for an adapted Web page. As mentioned in section 3.3 on Web page decomposition, the inter-object relationship (pre-order or in-order sequence) will be used to restore objects' presentation sequence and generate an adapted Web page. In-order sequence is used to describe parallel order of objects. For PC and NB, one object can be displayed beside or next to the other object in terms of spatial relationship, or one object can be rendered concurrently with the other object in terms of temporal relationship. In contrast, pre-order sequence is used to describe sequential order of objects. For smaller screen size of PDA and mobile phone, one object can be displayed above or below the other object in terms of spatial relationship, or one object can be rendered before or after the other object in terms of temporal relationship. Since devices with smaller screen size have smaller width; our principles rearrange the objects to remove the encumbrance of horizontal dragging. We move the object exceeding the boundary of screen width downward. From these steps, all objects will fit in the screen width and the results of adaptation appear as the following.

Figure 7 shows two groups of comparisons of Web page browsing between conventional and handheld devices without and with adaptation. Figure 7(a) shows NCU's BB login page (http://bb.ncu.edu.tw) on a desktop browser; Figure 7(b) shows the same login page on PDA screen without adaptation; Figure 7(c) shows the same login page on PDA screen with adaptation. Figure 7(d), 7(e), and 7(f) show NCU's BB system homepage on desktop screen, on PDA screen without adaptation, and on PDA screen with adaptation, respectively. The experimental results show that our adaptation mechanism is effective in transforming desktop-based Web page into PDA-based page with good reservation of semantic coherence.

\section{Experiments And Discussions}

To verify that the semantics of the adapted Web pages are equal to that of the original pages, we designed two experiments as follows:

Experiment 1: Ask 30 learning material designers each to build one learning Web page oriented to desktops. These 30 pages are then adapted using our content adaptation technique. Afterwards, the adapted pages are displayed on PDAs for their corresponding designers to observe any semantics variation. 


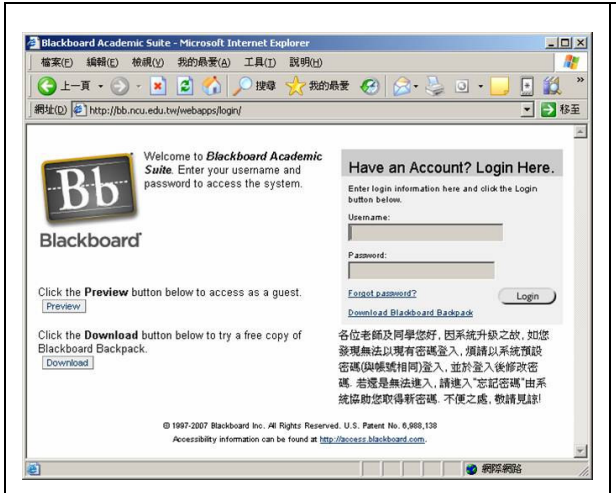

(a) desktop-based BB login page

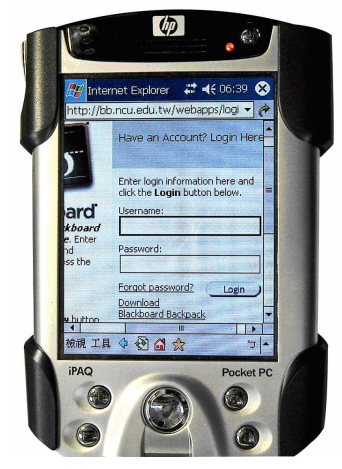

(b) PDA-based BB login page without adaptation

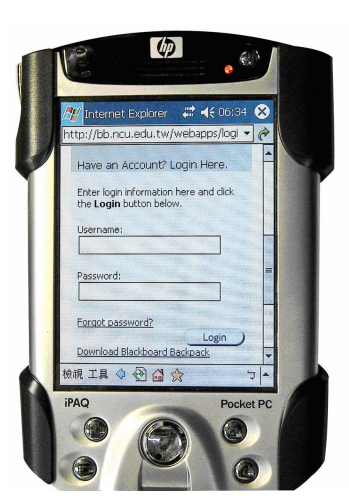

(c) PDA-based BB login page with adaptation

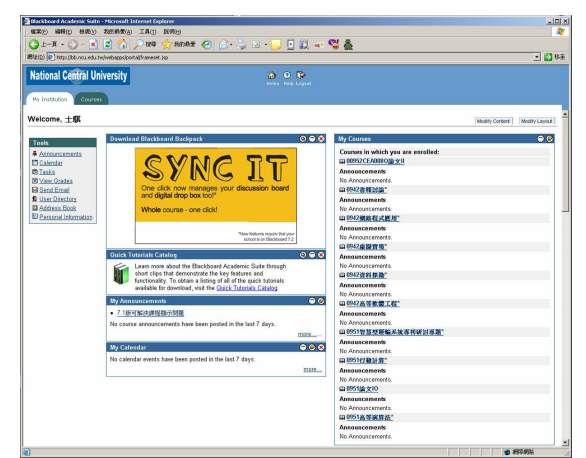

(d) desktop-based BB homepage

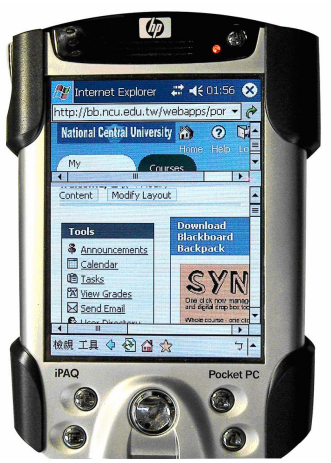

(e) PDA-based BB homepage without adaptation

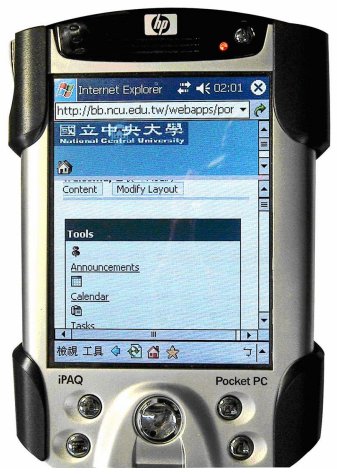

(f) PDA-based BB homepage with adaptation

Figure 7. Comparisons of BB login and homepages on desktop and PDA (adapted vs non-adapted). 
Experiment 2: Ask 60 students to browse the 30 adapted pages built in Experiment 1. Each page is assigned to two students to browse and report individually. Afterwards, we provide the corresponding original pages to each student and ask him/her to verify any semantics difference.

After the two experiments were conducted, we inquired the subjects about the semantics preservation between the adapted and original Web pages. We classified semantics preservation to five degrees, i.e., 100\% 91\%, 90\% 71\%, $70 \% \sim 51 \%$, $50 \% \sim 31 \%, 30 \% \sim 0 \%$. The degree of $100 \% \sim 91 \%$ means the semantics of the adapted and original pages are almost the same. On the contrary, the degree of 30\% 0\% is the worst situation, implying that the content adaptation messes up the organization of a page and leads to misunderstanding. The statistics are summarized in Table 4 and Table 5.

Table 4. Semantics preservation in Experiment 1.

\begin{tabular}{|c|c|c|c|c|c|}
\hline $\begin{array}{c}\text { Semantics } \\
\text { Preservation }\end{array}$ & $100 \%$ 91\% & $90 \% \sim 71 \%$ & $70 \% \sim 51 \%$ & $50 \% \sim 31 \%$ & $30 \% \sim 0 \%$ \\
\hline $\begin{array}{c}\text { Subjects } \\
\text { (30 designers) }\end{array}$ & 26 & 4 & 0 & 0 & 0 \\
\hline
\end{tabular}

Table 5. Semantics preservation in Experiment 2.

\begin{tabular}{|c|c|c|c|c|c|}
\hline $\begin{array}{c}\text { Semantics } \\
\text { Preservation }\end{array}$ & $100 \% \sim 91 \%$ & $90 \% \sim 71 \%$ & $70 \% \sim 51 \%$ & $50 \% \sim 31 \%$ & $30 \% \sim 0 \%$ \\
\hline $\begin{array}{c}\text { Subjects } \\
(60 \text { students })\end{array}$ & 55 & 4 & 1 & 0 & 0 \\
\hline
\end{tabular}

The above semantics preservation statistics have demonstrated that our content adaptation technique is effective in analyzing the semantics from original Web pages and in keeping semantics coherence for adapted web pages.

We also designed another experiment to evaluate whether our Web page adaptation mechanism met the two objectives addressed in the Introduction section - to remove the time limitation by encouraging students to use BB besides class hours, and to remove the space limitation by encouraging students to use BB out of classroom. This experiment also considered the situation when Web page adaptation might result in degradation of content quality that may affect students' satisfactions level on the adapted pages.

Without losing generality, we randomly selected one course from the 529 BB courses as the test bed of the experiment. 43 students enrolled in a course, titled "XML-based Web Service and Applications," participated in this experiment. The performance comparison was measured by the percentage of students who used BB before and one month after we applied page adaptation. The results are summarized in Table 6. 
Table 6. Comparison of BB usage before and after applying page adaptation.

\begin{tabular}{lll}
\hline & Percentage of students who use BB \\
\hline Time slot & Before applying adaptation & $\begin{array}{l}\text { One month after applying } \\
\text { adaptation }\end{array}$ \\
\hline Before class & $12 \%$ & $23 \%$ \\
After class & $3 \%$ & $14 \%$ \\
\hline Location & Without adaptation & With adaptation \\
\hline Outdoor & $0 \%$ & $16 \%$ \\
On the move & $0 \%$ & $2 \%$ \\
\hline BB area & Without adaptation & With adaptation \\
\hline Announcement & $4 \%$ & $7 \%$ \\
Course & $74 \%$ & $52 \%$ \\
documents & & \\
Communication & $6 \%$ & $20 \%$ \\
Discussion board & $12 \%$ & $18 \%$
\end{tabular}

Table 6 reveals several findings worth noting. First, page adaptation increases flexibility in access time and access space. Applying page adaptation to BB encourages students to use BB both before they attend class (increased from $12 \%$ to $23 \%$ ) and after they are dismissed from class (increased from 3\% to 14\%). Second, Content adaptation also increases effective learning content access from outdoor (increased from $0 \%$ to $16 \%$ ) and on the move (increased from $0 \%$ to $2 \%$ ).

Third, applying page adaptation increases students' usage of various collaboration supporting tools in BB (e.g., announcement area increased from $4 \%$ to $7 \%$, communication area increased from $6 \%$ to $20 \%$, and discussion board increased from $12 \%$ to $18 \%$ ). This phenomenon shows that students are willing to continue collaboration while they are staying outdoors or on the move.

We further designed a qualitative evaluation to test students' satisfaction level with the adapted content appeared in the adapted page. The motivation is that we realize adaptation might result in degradation of Web page quality, which may affect students' satisfaction with the adapted content. The reasons of quality degradation might include: reading difficulties due to either a device's screen size or improper content resizing or page reorganization, or degradation in fidelity due to either devices' computing and bandwidth limitation or improper transcoding. Thus, we designed three evaluation questions to test students' satisfaction regarding images, texts, and hyperlinks appeared in adapted pages.

(1) Are students satisfied with the quality of adapted images?

(2) Are students satisfied with the quality of adapted text?

(3) Are students satisfied with the navigation quality of the adapted page? 
All students in the aforementioned class participated in this evaluation survey. Each student was asked to use PDAs and mobile phones to access the aforementioned three sections of any typical BB page (Course home, Announcement, and Course document) with page adaptation, and then record their answers to the three questions above. The reason why we selected the three sections is their significant delivery features. The Course home section typically has a relatively stable structure; the Announcement section is frequently updated so that its structure is volatile; and the Course document section contains various kinds of multimedia presentation objects. In short, we used BB's diverse content types as the test bed to evaluate students' satisfaction of adapted pages.

We summarized our findings from the students' responses in Table 7. When students use PDAs, the Course home section provides the best results of content adaptation because it has more stable content page structures; $83 \%$ of students are satisfied with the adapted images on a Course home section. Regarding texts and hyperlinks on the Home section, we found that most of the students are satisfied with the adapted results. $96 \%$ and 94\% of students are satisfied with the adapted text and hyperlinks, respectively. These are the two highest comprehensions in this evaluation experiment.

Table 7. Evaluation of satisfaction of various content types on three different BB sections

\begin{tabular}{llll}
\hline $\begin{array}{l}\text { Blackboard } \\
\text { page }\end{array}$ & Adapted content & With PDA (\%) & With phone (\%) \\
\hline Course home & image & $83 \%$ & $67 \%$ \\
& text & $96 \%$ & $87 \%$ \\
& $\begin{array}{l}\text { Navigation } \\
\text { (hyperlinks) }\end{array}$ & $94 \%$ & $85 \%$ \\
\hline Announcement & image & $68 \%$ & $47 \%$ \\
& text & $75 \%$ & $78 \%$ \\
& $\begin{array}{l}\text { Navigation } \\
\text { (hyperlinks) }\end{array}$ & $71 \%$ & $72 \%$ \\
\hline Course & image & $80 \%$ & $60 \%$ \\
document & text & $86 \%$ & $81 \%$ \\
& Navigation & $85 \%$ & $77 \%$ \\
\hline
\end{tabular}

However, when the students use mobile phones, they oftentimes are unsatisfied with the adapted content due to smaller screen size and less computing capability, especially on adapted images appeared on the Announcement section due to its nature of frequent changes. Only $47 \%$ of the students said that they are satisfied with the adapted images. This number is the lowest satisfaction rate in this experiment. 
The students also expressed their strong demands of Web page adaptation when they browsed image-intensive materials such as the sections of Course document. They indicated that a flexible and readable content presentation is one of their major concerns when they use handheld devices for learning. Table 8 is a summary of the students' experience of using BB equipped with our Web page adaptation technology.

Table 8. Summary of students' experience of using BB equipped with our Web page adaptation technology.

No. Students' experience

$1 \quad 18 \%$ students claimed that they would use mobile devices to browse BB course materials either outside of classroom or on the move.

$212 \%$ students claimed that they would use mobile devices to check messages on the Communication and Discussion Board to keep in touch with their classmates.

$37 \%$ students claimed that they would use mobile devices to check the Announcement on BB within one hour before classes begin.

$44 \%$ students claimed that they would use mobile devices to check the Announcement on BB to ensure they do not miss any assignment within one hour after classes end.

$52 \%$ students claimed that they would use mobile devices to browse Course documents on $\mathrm{BB}$ to preview course materials one hour before classes begin.

6 No students expressed that they would use mobile devices to browse the Course documents on BB to review course materials.

Finally, we conducted a survey regarding how Web page adaptation affects the usage of portable devices. The mean was computed based on five-point Likert scale. The result is shown in Table 9. We found that most of the students consider page adaptation important, and they are satisfied with the results of adaptation even though the quality of presentation may be degraded due to limitations of computing environments such as network and device constraints. Most of the students complained about the waiting time of adaptation, which discouraged their interests in using adaptation. Nevertheless, most of the students are willing to sacrifice waiting time for better browsing experience. Regarding the quality of adaptation, we found that most of the students agree that the results of adaptation meet their preferences and accessibility. This is because we explicitly ask the students to fill out their preferences and accessibility so that we can provide better match to their needs. Users are not satisfied with the quality of adaptation regarding their situations. This is because our current system does not take into consideration users' residing contexts. This can be one of our future research topics.

The students also provide some further inputs about their expectations. One is that they prefer simplicity of user interface; the other is that they expect us to maintain rationality of Web page semantics. 
Table 9. Performance of Web page adaptation on handheld devices

\begin{tabular}{|c|c|c|c|}
\hline No. & Questionnaire & Mean & SD \\
\hline 1 & $\begin{array}{l}\text { Do you think page adaptation improves your browsing } \\
\text { experience on portable devices? }\end{array}$ & 3.89 & 1.24 \\
\hline 2 & $\begin{array}{l}\text { Are you satisfied with the waiting time due to page } \\
\text { adaptation? }\end{array}$ & 1.25 & 0.56 \\
\hline 3 & $\begin{array}{l}\text { Will you sacrifice waiting time for better browsing } \\
\text { experience on portable devices? }\end{array}$ & 3.43 & 1.45 \\
\hline 4 & $\begin{array}{l}\text { Does page adaptation increase your interests of using } \\
\text { portable devices? }\end{array}$ & 4.23 & 0.78 \\
\hline 5 & $\begin{array}{l}\text { Do you think portable devices should provide the same } \\
\text { presentation quality as desktops? }\end{array}$ & 3.92 & 1.14 \\
\hline 6 & $\begin{array}{l}\text { Is it acceptable to gracefully degrade the quality of content } \\
\text { due to page adaptation? }\end{array}$ & 4.17 & 0.86 \\
\hline 7 & Does page adaptation meet your accessibility? & 3.75 & 0.92 \\
\hline 8 & Does page adapt & 3.67 & 0.87 \\
\hline 9 & s page adaptation & 2.94 & 1.24 \\
\hline 10 & Does page adaptation meet your network constraints? & 3.17 & 1.17 \\
\hline 11 & Does page adaptation meet your device constraints? & 3.34 & 0.94 \\
\hline
\end{tabular}

Meanwhile, we aim to extend the time and space of learning while encouraging and facilitating learning collaboration among students. By applying mobile learning and Web page adaptation techniques, we intend to (1) remove the time limitation by encouraging students to use BB before they attend classes and after they are dismissed from classes, (2) to eliminate the space limitation by encouraging students to use BB outside of classroom, and (3) to encourage students to collaborate by providing collaboration tools.

\section{Conclusions}

Based on our experimental results, we conclude that our self-adaptive Web content adaptation technique helps to attain the goal of presentation optimization by using minimal information to represent maximized meaning of content. In this paper, we have presented our content adaptation technique and applied it to improve mobile learning in the Blackboard Learning System. Without updating any previous course content designed for desktop computers, our solution realizes a "One-for-All" feature. The hope of "Write Once, Show Everywhere" allows content providers to prepare a content page only once in HTML oriented to desktop computers; and it can be presented onto various devices with the help of our content adaptation technique. Our experimental results show that content adaptation can dramatically reduce the access time to course content, especially when students are using handheld devices. We believe that when portable devices become more popular in student communities, our content adaptation will have more impacts on the enhancement of mobile learning. 
According to market surveys, learning materials might have various structures, constraints and forms. There are several popular elements for Web page building, such as FLASH, Ajax, and MOD. These techniques richen user experience and improve mobile learning with more powerful interactions. This study focuses on dealing with Web pages in HTML-based documents at present. We plan to extend our content adaptation technique to handle other formats of Web pages in the future.

Although our work was conducted on the platform of Blackboard Leaning System, it can be applied to any other platform. In general, our self-adaptive content adaptation technique can be implemented either as an individual Web service or a plug-in to a Web browser. In other words, it can be used either as an intermediate server or a downloadable on client side.

\section{Acknowledgments}

This work is supported by National Science Council, Taiwan under grants NSC-95-2520S-008-006-MY3 and NSC-96-2628-S-008-008-MY3.

\section{References}

AL-Bastaki, Y. \& Al-Ajeeli, A. (2005). A framework for a WAP-based course registration system. Computers \& Education, 44, 327-342.

$\begin{array}{llll}\text { Blackboard.com. } & \text { Blackboard } & \text { learning } & \text { system. from }\end{array}$ http://www.blackboard.com/products/academic_suite/learning_system/index.

Chang, C.K., Wang, C. Y., \& Chen, G.D. (2006). Ubiquitous learning Website: scaffold learners by mobile devices with information-aware techniques. Computers \& Education.

Markett, C., Sa'nchez, I.A., Weber, S. \& Tangney, B. (2006). Using short message service to encourage interactivity in the classroom. Computers \& Education, 46, 280-293.

Segall, N., Doolen, T.L., \& Porter, J.D (2005). A usability comparison of PDA-based quizzes and paper-and-pencil quizzes. Computers \& Education, 45, 417-432.

Virvou, M. \& Alepis, E. (2005). Mobile educational features in authoring tools for personalised tutoring. Computers \& Education, 44, 53-68.

W3C. Document Object Model (DOM). from http://www.w3.org/DOM/.

Yang, S.J.H. and Shao, \& N.W.Y. (2006). An ontology based content model for intelligent Web content access services. International Journal of Web Service Research (JWSR), 3(2), 5978.

Yang, S.J.H., Zhang, J., \& Chen, I.Y.L. (2007a). A JESS enabled context elicitation system for providing context-aware Web services. Expert Systems with Applications, 34(4), 22542266.

Yang, S.J.H., Zhang, J.\& Chen, I.Y.L. (2007b). Ubiquitous provision of context-aware Web services. International Journal of Web Services Research (JWSR), 4(4), 83-103. 
Yang, S.J.H., Zhang, J., Shao, N.W.Y., \& Chen, R.C.S. (2007c). A UOI-Based Content Adaptation Method for Improving Web Content Accessibility in the Mobile Internet. ETRI Journal, 29(6), 794-807.

Zurita, G. \& Nussbaum, M. (2004). Computer supported collaborative learning using wirelessly interconnected handheld computers. Computers \& Education, 42, 289-314. 